\title{
Article \\ Protein Kinase A (PRKA) Activity Is Regulated by the
Proteasome at the Onset of Human Sperm Capacitation
}

\author{
Héctor Zapata-Carmona ${ }^{1}$ (D), Lina Barón ${ }^{1}$, Milene Kong ${ }^{1}$ and Patricio Morales ${ }^{1,2, *(D)}$ \\ 1 Laboratorio de Biología de la Reproducción, Facultad de Ciencias de la Salud, Departamento Biomédico, \\ Universidad de Antofagasta, Antofagasta 1240000, Chile; hector.zapata@uantof.cl (H.Z.-C.); \\ lina.baron@uantof.cl (L.B.); milene.kong@uantof.cl (M.K.) \\ 2 Instituto Antofagasta, Universidad de Antofagasta, Antofagasta 1240000, Chile \\ * Correspondence: patricio.morales@uantof.cl
}

Citation: Zapata-Carmona, H.; Barón, L.; Kong, M.; Morales, P. Protein Kinase A (PRKA) Activity Is Regulated by the Proteasome at the Onset of Human Sperm Capacitation. Cells 2021, 10, 3501. https://doi.org/ 10.3390/cells10123501

Academic Editors: Michal Zigo and Peter Sutovsky

Received: 1 November 2021

Accepted: 7 December 2021

Published: 11 December 2021

Publisher's Note: MDPI stays neutral with regard to jurisdictional claims in published maps and institutional affiliations.

Copyright: (c) 2021 by the authors. Licensee MDPI, Basel, Switzerland. This article is an open access article distributed under the terms and conditions of the Creative Commons Attribution (CC BY) license (https:// creativecommons.org/licenses/by/ $4.0 /)$.

\begin{abstract}
The proteasome increases its activity at the onset of sperm capacitation due to the action of the SACY/PRKACA pathway; this increase is required for capacitation to progress. PRKA activity also increases and remains high during capacitation. However, intracellular levels of cAMP decrease in this process. Our goal was to evaluate the role of the proteasome in regulating PRKA activity once capacitation has started. Viable human sperm were incubated in the presence and absence of epoxomicin or with $0.1 \%$ DMSO. The activity of PRKA; the phosphorylation pattern of PRKA substrates (pPRKAs); and the expression of PRKAR1, PRKAR2, and AKAP3 were evaluated by Western blot. The localization of pPRKAs, PRKAR1, PRKAR2, and AKAP3 was evaluated by immunofluorescence. Treatment with epoxomicin changed the localization and phosphorylation pattern and decreased the percentage of PPRKAs-positive sperm. PRKA activity significantly increased at $1 \mathrm{~min}$ of capacitation and remained high throughout the incubation. However, epoxomicin treatment significantly decreased PRKA activity after $30 \mathrm{~min}$. In addition, PRKAR1 and AKAP3 were degraded by the proteasome but with a different temporal kinetic. Our results suggest that PRKAR1 is the target of PRKA regulation by the proteasome.
\end{abstract}

Keywords: sperm; capacitation; proteasome; protein kinase A; PRKA regulatory subunits; AKAP3

\section{Introduction}

The ubiquitin-proteasome system (UPS) is a major pathway for intracellular protein degradation [1,2]. In this system, substrates are typically marked for degradation by covalent linkages to multiple ubiquitin molecules. Ubiquitin is a monomeric protein of 76 amino acid residues and has a molecular weight of $8.5 \mathrm{kDa}[3,4]$; it is an essential element of the UPS [5]. Once marked by polyubiquitin chains, proteins are rapidly degraded by the proteasome. Proteasome function is crucial for cellular viability [6,7]. The core of the proteasome, also called 205 proteasome, is formed by two pairs of homologous rings, each containing seven subunits. The two outer rings contain $\alpha$-type subunits (PSMA1-7), the function of which is to operate as a "gate" through which proteins enter the catalytic sites [8]. The $\beta$-subunits (PSMB1-7) form the two inner rings. Three of the $\beta$-subunits, $\beta 1$ (PSMB6), $\beta 2$ (PSMB7), and $\beta 5$ (PSMB5), are catalytically active and possess caspase-like (or peptidylglutamyl-peptide), trypsin-like, and chymotrypsin-like activities, respectively $[2,9,10]$. Proteasomes are not static complexes, and the activity of the $20 \mathrm{~S}$ proteasome is modulated by the binding of different regulatory complexes: $195, \mathrm{PA} 28 \alpha / \beta$, PA28 $\gamma$, and PA200 [8,11,12].

Proteasomes are present in the sperm of numerous species, and several studies have shown that human sperm have all the machinery of the UPS [13-16]. More recent studies have provided compelling evidence of UPS being a regulating element of sperm capacitation [16-18]. Sperm capacitation was first independently reported over seven decades ago by Austin [19] and Chang [20]. This phenomenon is a process that occurs in the oviduct 
of the female reproductive tract, but it can be mimicked in vitro using chemically defined media. These media are based on the concentration of electrolytes and macromolecules present in the oviductal fluid. A recent study showed that oviductal fluid can modulate capacitation-associated events in vitro [21]. During the process of capacitation there are cholesterol efflux from the sperm plasma membrane and increased influx of bicarbonate and $\mathrm{Ca}^{2+}$, which activate soluble adenyl cyclase (SACY) to produce cAMP, leading to protein kinase A (PRKA) activation, protein tyrosine phosphorylation, actin polymerization, and the development of hyperactivated motility [22]. PRKA is a tetrameric serine/threonine (Ser/Thr) kinase holoenzyme composed of two regulatory (R) and two catalytic subunits (C). Mice sperm that lack the unique PRKA catalytic subunit $C \alpha 2$ are infertile, despite normal mating behavior, and do not show the increase in tyrosine phosphorylation after sperm incubation in a capacitating medium [23], suggesting the involvement of PRKA in sperm capacitation. PRKA activity depends on the binding of four cAMP molecules to the regulatory subunits (two molecules per subunit) that allows tetramer dissociation and activation of the catalytic subunits [23]. Once free and active, the catalytic subunits phosphorylate a wide variety of substrates in the Arg-X-X-Ser/Thr motif [24,25].

In human spermatozoa, reports indicate that the increase in cAMP levels and activation of PRKA are among the first intracellular events of capacitation since cells incubated in a capacitating medium show a rapid ( $\leq 1 \mathrm{~min}$ ) increase in PRKA activity, and this activity remains high and constant during this process [26,27]. This activation of PRKA leads to increased Ser/Thr protein phosphorylation, which will ultimately lead to sperm capacitation. However, intracellular levels of cAMP begin to decrease significantly after $1 \mathrm{~min}$, reaching values similar to those observed in spermatozoa incubated in non-capacitating conditions at $60 \mathrm{~min}$ [28]. We recently described that one of the substrates of PRKACA during early stages of human sperm capacitation is the proteasome [16]. We showed that proteasome activity is directly regulated by the SACY/cAMP/PRKACA pathway. The chymotrypsinlike activity of the sperm proteasome significantly increased after $5 \mathrm{~min}$ of capacitation and remained high during $60 \mathrm{~min}$ incubation. Treatment with PRKA inhibitors significantly decreased proteasome activity. PRKA is located in the same subcellular compartments as the proteasome and co-immunoprecipitates with the proteasome. Furthermore, treatment with the proteasome-specific inhibitor epoxomicin significantly blocked capacitation and decreased the increase in tyrosine phosphorylation during capacitation. These data suggests that there might be a feedback regulation between PRKA and the proteasome, where at early incubation times PRKA phosphorylates and activates the proteasome, while at later times the proteasome regulates PRKA activity. Therefore, the aim of the present work was to evaluate the role of the sperm proteasome in regulating PRKA activity once human sperm capacitation has started.

\section{Materials and Methods}

\subsection{Ethics Statement}

The research presented in this manuscript was approved by the Ethics Committee on Scientific Research of the University of Antofagasta (CEIC-UA). The Ethics Committee approved the use of all human semen samples described in this study, and we followed the current guidelines for human semen studies [29]. All donors, anonymous to the researchers, signed a consent form for the use of their spermatozoa for research purposes.

\subsection{Chemicals and Reagents}

The following reagents were purchased from Sigma Chemical Co. (St. Louis, MO, USA): N $\alpha$-tosyl-L-lysine chloromethyl ketone hydrochloride (TLCK); bovine serum albumin (BSA; A7030); Ponceau Red; HEPES; ethylenediaminetetraacetic acid (EDTA); sodium chloride $(\mathrm{NaCl})$; potassium chloride $(\mathrm{KCl})$; sodium phosphate dibasic $\left(\mathrm{Na}_{2} \mathrm{HPO}_{4}\right)$; potassium phosphate monobasic $\left(\mathrm{KH}_{2} \mathrm{PO}_{4}\right)$; calcium chloride $\left(\mathrm{CaCl}_{2}\right)$; magnesium chloride $\left(\mathrm{MgCl}_{2}\right)$; magnesium sulfate $\left(\mathrm{MgSO}_{4}\right)$; sodium bicarbonate $\left(\mathrm{NaHCO}_{3}\right)$; tris hydrochloride (Tris-HCl); Tween 20; sodium dodecyl sulfate (SDS); $\beta$-mercaptoethanol; glycerol; ammo- 
nium persulfate; N'-Tetramethylethylenediamine (TEMED); Percoll; Hoechst 33258 (H258); 1,4-diazabicyclo [2.2.2.] octane (DABCO); dimethyl sulfoxide (DMSO); sodium orthovanadate $\left(\mathrm{Na}_{3} \mathrm{VO}_{4}\right)$; sodium fluoride $(\mathrm{NaF})$; phenylmethylsulfonyl fluoride (PMSF); aprotinin; and leupeptin. The following compounds were purchased from Enzo Life Sciences (Farmingdale, NY, USA): $\mathrm{N}$-[2-(p bromocinnamylamino) ethyl]-5-isoquinolinesulfonamide $2 \mathrm{HCl}$ (H89) and $\mathrm{N}$-Acetyl- $N$-methyl-L-isoleucyl-L-isoleucyl- $N$-[(1S)-3-methyl-1-[[(2R)-2 methyloxiranyl]carbonyl]butyl]-L-threoninamide (epoxomicin). Dako Fluorescent Mounting Medium was purchased from Dako North America.

The deionized water used in these experiments was purified to 18 megohms with an EASY-pure UV/UF ion-exchange system (Barnstead/Thermolyne, Dubuque, IA, USA). All other chemicals were of analytical grade and obtained from standard sources.

\subsection{Culture Media}

The basic medium used for all the experiments was a modified Tyrode's medium lacking BSA and $\mathrm{NaHCO}_{3}$, as described [16]. This medium was designated non-capacitating medium (NCM). The capacitating medium (CM) was similar to NCM, except that it was supplemented with $2.6 \% \mathrm{BSA}$ and $25 \mathrm{mM} \mathrm{NaHCO}_{3}$ [16]. The $\mathrm{pH}$ of all media was adjusted to $7.4-7.45$ before use.

\subsection{Semen Collection}

A total of 27 semen samples were provided by healthy donors aged between 20 and 30 years; some donors provided more than one sample. Freshly ejaculated spermatozoa were obtained by masturbation after 2-3 days of sexual abstinence. Samples obtained from the same donor were requested with at least a 2-week interval. Semen was collected in a sterile vessel, and each lot was tested for sperm toxicity. All semen samples used were normospermic according to World Health Organization criteria [30]. Semen samples were allowed to liquefy for $30-60 \mathrm{~min}$ at $37^{\circ} \mathrm{C}$. All semen samples were processed by the same person using compatible equipment, and analyses of volume, $\mathrm{pH}$, sperm concentration, and percentages of motile and viable spermatozoa were performed. The mean values for semen parameters are summarized in Table S1 (Supplementary Materials S1).

\subsection{Sperm Selection}

Motile sperm were obtained using a dual Percoll gradient (40/80\%), as described previously [15]. The final sperm pellet was resuspended in the appropriate medium at the required concentration. Approximately $5 \times 10^{6}$ spermatozoa $/ \mathrm{mL}$ were incubated for $0(\mathrm{NCM}), 1,10,15,30$, and $60 \mathrm{~min}(\mathrm{CM})$ at $37^{\circ} \mathrm{C}$ and $5 \% \mathrm{CO}_{2}$ in air and in the presence or absence of inhibitors. None of the inhibitors or solvents used in this study negatively affected sperm viability or motility (data not shown).

\subsection{SDS-PAGE and Immunoblotting}

After capacitation, sperm aliquots were washed twice in $1 \mathrm{~mL}$ of cold phosphatebuffered saline (PBS) $\left(137 \mathrm{mM} \mathrm{NaCl}, 2.7 \mathrm{mM} \mathrm{KCl}, 1.5 \mathrm{mM} \mathrm{KH}_{2} \mathrm{PO}_{4}, 4.3 \mathrm{mM} \mathrm{Na} 2 \mathrm{PO}_{4}\right.$, $\mathrm{pH}$ 7.4) and centrifuged at $9000 \times g$ for $30 \mathrm{~s}$. The pellet was resuspended in Laemmli sample buffer [31], boiled for $5 \mathrm{~min}$, and centrifuged once more. Supernatants were then supplemented with $5 \% \beta$-mercaptoethanol and boiled again for $5 \mathrm{~min}$. Isolated proteins were separated on SDS-PAGE $(60 \mathrm{~mA}, 120 \mathrm{~min})$. Afterwards, proteins were transferred to polyvinylidene fluoride (PVDF) membranes at $250 \mathrm{~mA}$ for $60 \mathrm{~min}$ at $4{ }^{\circ} \mathrm{C}$. Transfer was monitored by Ponceau red stain. The membranes were blocked with $5 \%$ milk in TRIS-buffer saline with $0.1 \%$ Tween 20 (T-TBS) and then incubated with the primary antibodies anti phospho-PRKA substrates (pPRKAs) (Cell Signaling Technologies, Danvers, MA, USA. \#9624); total PRKAC (Cell Signaling Technologies, \#4782); phospho-PRKAC (Thr197) (Cell Signaling Technologies, \#4781); PRKAR1 (Cell Signaling Technologies, \#3927); PRKAR2 (Epitomics, Burlingame, CA, USA. \#1528-1); and AKAP3 (Santa Cruz Biotechnology, Dallas, TX, USA. \#SC-135146). Membranes were incubated overnight at $4{ }^{\circ} \mathrm{C}$ with gentle shaking. 
After this step, membranes were washed six times and incubated with the appropriate biotinylated secondary antibody for $1 \mathrm{~h}$ at room temperature. Next, the membranes were washed with T-TBS for the last time, and blots were visualized by chemiluminescence (Amersham Corp., Sydney, Australia) according to the manufacturer's instructions. Finally, the signal was imaged using an In-Vivo F Pro molecular imaging system (Bruker Corporation, Billerica, MA, USA).

\subsection{Stripping the PVDF Membranes}

To confirm equal protein load, blots were stripped and re-probed with an antibody against $\beta$-tubulin (Developmental Studies Hybridoma Bank, Iowa City, IA, USA. \#AB2315513). For this procedure, $15 \mathrm{~mL}$ of stripping buffer, consisting of $2 \%(w / v) \mathrm{SDS}, 62.5 \mathrm{mM}$ Tris, pH 6.7, and $100 \mathrm{mM}$ 2-mercaptoethanol, was added to the membrane for $1 \mathrm{~h}$ with constant shaking at $60{ }^{\circ} \mathrm{C}$. The membrane was then washed 6 times for $10 \mathrm{~min}$ in TBS, blocked, and probed with the primary antibody, as described above.

\subsection{Immunofluorescence Assessment of Spermatozoa}

Spermatozoa were fixed in $4 \%$ paraformaldehyde for $15 \mathrm{~min}$ and washed twice by centrifugation with $0.1 \mathrm{M}$ glycine in PBS at $14,000 \times g$ for $2 \mathrm{~min}$. Then, the cells were permeabilized for 10 min with $0.1 \%$ Triton X-100 diluted in PBS and washed twice with PBS at $14,000 \times g$ for $2 \mathrm{~min}$. The final pellet was resuspended in PBS supplemented with $1 \%$ BSA for $30 \mathrm{~min}$. The sperm suspensions were spread on a glass slide and were allowed to dry for $15 \mathrm{~min}$ at room temperature. Then, the samples were incubated overnight with the primary antibodies for pPRKAs (dilution 1:50), PRKAR1 (dilution 1:20), PRKAR2 (dilution 1:100), and AKAP3 (dilution 1:20). Samples were washed 3 times with PBS and incubated with chicken anti-rabbit conjugated to Alexa 594 antibody (Thermo Fisher Scientific, Waltham, MA, USA. \#A-21442) or goat anti-mouse conjugated to Alexa 488 antibody (Thermo Fisher Scientific, \#A-10680) diluted 1:500 in 1\% BSA-PBS for $2 \mathrm{~h}$ at room temperature. Finally, the samples were washed 3 times for 5 min with PBS, and the coverslips were mounted with Dako Fluorescent Mounting Medium and examined under a confocal microscope (Leica TCS SP8 SMD, Mannheim, Germany). A minimum of 400 cells were analyzed in each sample. Controls using a secondary antibody alone were performed to assure specificity.

\subsection{Statistical Analyses}

Data were assessed by the Kolmogorov-Smirnov test and were normally distributed. They were analyzed by a one-way analysis of variance with Tukey's post hoc test. Densitometry analysis was performed using the image $2.0 \mathrm{j}$ software and normalized with regard to the internal control tubulin.

Quantitative results are presented as mean values and SEM. In all cases, results with $p<0.05$ were considered as values with significant differences.

\section{Results}

\subsection{The Proteasome Is Required for PRKA Signaling during Human Sperm Capacitation}

The activation of PRKA during capacitation has been widely studied, and it has been defined as a fundamental event for sperm to acquire its fertilizing capacity. In this study, PRKA activity was evidenced by the phosphorylation of its catalytic subunit Thr197 [32-34] (Figure 1A) and specific presence of pPRKAs by Western blot (Figure 2). The results show that the intensity of the phospho PRKAC band of sperm incubated in NCM (0 min) was lower than those incubated in CM (Figure 1A, $p<0.01$ ). In sperm incubated for $1 \mathrm{~min}$ in $C M$, a rapid increase in the intensity of the phospho PRKAC band was observed. The intensity of this band remained high and constant for the next $60 \mathrm{~min}$. In the presence of the proteasome inhibitor epoxomicin, the intensity of the phospho PRKAC band was unaffected for the first $15 \mathrm{~min}$. However, sperm incubated for 30 and $60 \mathrm{~min}$ in the presence of epoxomicin exhibited a significant decrease in the intensity of the phospho PRKAC band $(p<0.01)$ compared to the controls without inhibitor. As expected, when sperm 
were incubated for $60 \mathrm{~min}$ in CM in the presence of the PRKA inhibitor, H89, a significant decrease in the intensity of the phospho PRKAC band was observed (Figure 1).
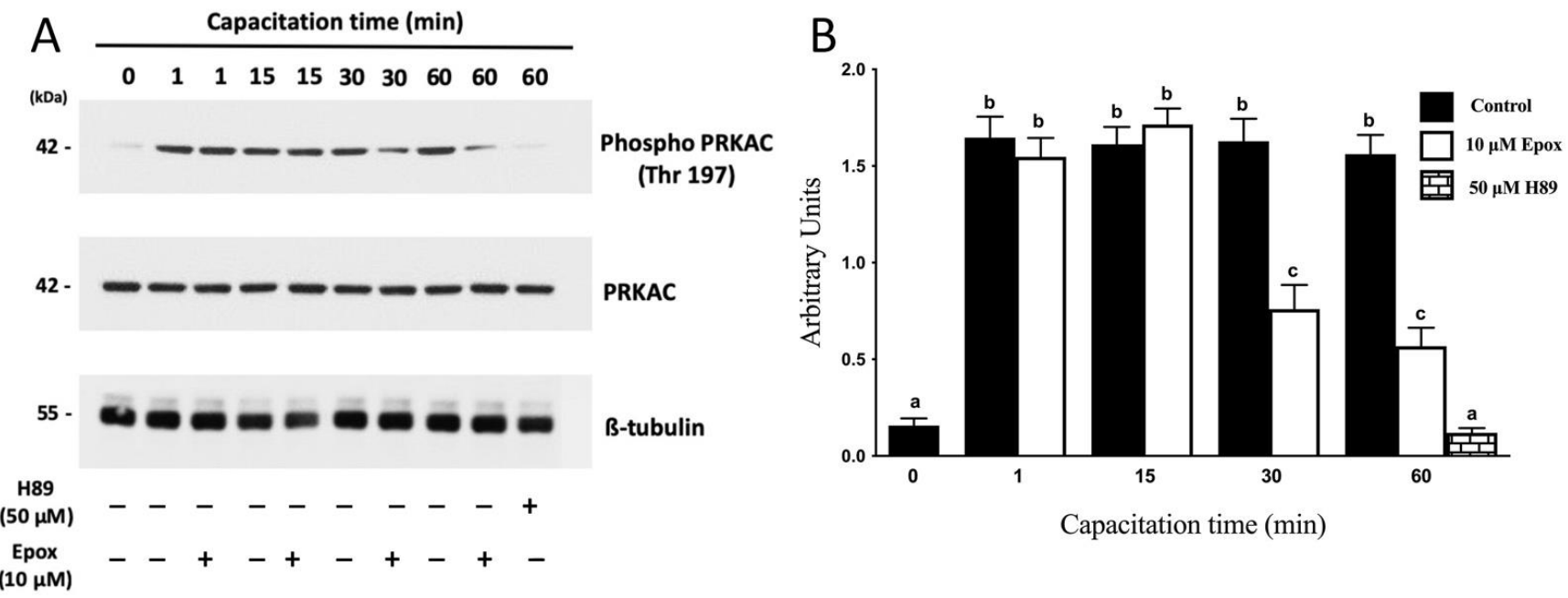

Figure 1. Effect of proteasome inhibition on the phosphorylation of PRKAC in Thr197 during human sperm capacitation. Human sperm were incubated for different times with $0.1 \%$ DMSO, $10 \mu \mathrm{M}$ epoxomicin, or with $50 \mu \mathrm{M}$ H89. Time 0 (0 min) corresponds to sperm incubated in non-capacitating conditions. (A) Western blot of PRKA activity evaluated using the anti-phospho Thr197 antibody against the catalytic subunit of PRKA (PKA-C). (B) For the densitometric analysis, total PRKAC/ B-tubulin levels were used as loading control. Bars represent the mean \pm S.E.M of five different experiments. Different letters indicate statistically significant differences $(p<0.01)$ between groups.
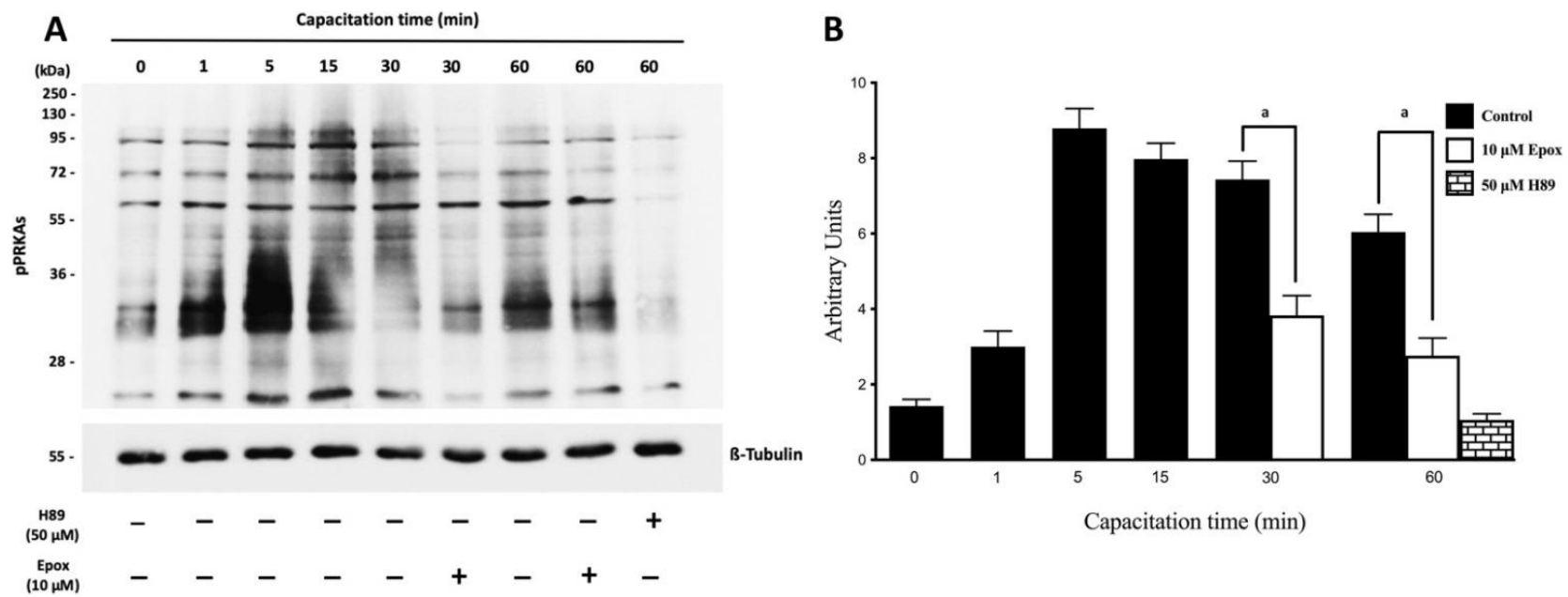

Figure 2. Effect of proteasome inhibition on the phosphorylation of PRKA substrates (pPRKAs) during human sperm capacitation. Human sperm were incubated for different times with $0.1 \%$ DMSO, $10 \mu \mathrm{M}$ epoxomicin, or with $50 \mu \mathrm{M}$ H89. The time 0 ( $0 \mathrm{~min}$ ) corresponds to sperm incubated in non-capacitating conditions. (A) Phosphorylated PRKA substrates (pPRKAs) were evaluated by Western blot. (B) For the densitometric analysis, $\beta$-tubulin was used as loading control. Bars represent mean \pm S.E.M. of seven different experiments. a indicates $p<0.001$ vs. corresponding control.

In the next experiments, PRKA activity and its regulation by the proteasome was evidenced by the appearance of pPRKAs and the effect of epoxomicin, respectively. Sperm incubated in NCM showed low levels of pPRKAs (Figure 2A, T0 min). Sperm cells incubated in CM showed high levels of pPRKAs, starting at $1 \mathrm{~min}$ and remaining high for the next $60 \mathrm{~min}$. In contrast, sperm incubated for 30 and $60 \mathrm{~min}$ in the presence of epoxomicin showed a significant decrease in the intensity of the pPRKAs bands (Figure 2A, $p<0.001$ ).

The next experiment was designed to determine the effect of epoxomicin on the subcellular localization of pPRKAs in human sperm (Figure 3). Sperm incubated in NCM 
(T0) presented pPRKAs labeled exclusively in the flagellum (Figure 3B with less intensity and a lower percentage than in sperm incubated in CM for $60 \mathrm{~min}$ (T60, Figure 3F) (65 \pm 9\% vs. $91 \pm 2 \%$, respectively. $p<0.01$ ). Although most of the sperm incubated in CM for $60 \mathrm{~min}$ presented labeling in the flagellum, only $24 \pm 6 \%$ had labeling in the equatorial segment of the head (Figure 3F). Sperm incubated for $60 \mathrm{~min}$ in the presence of epoxomicin (T60 + Epox, Figure $3 \mathrm{~J})$, presented labeling on the flagellum $(86 \pm 8 \%)$ similar to that observed in the control without inhibitor and with the same fluorescence intensity. However, these sperm did not show labeling in the equatorial segment of the head (Figure 3J).
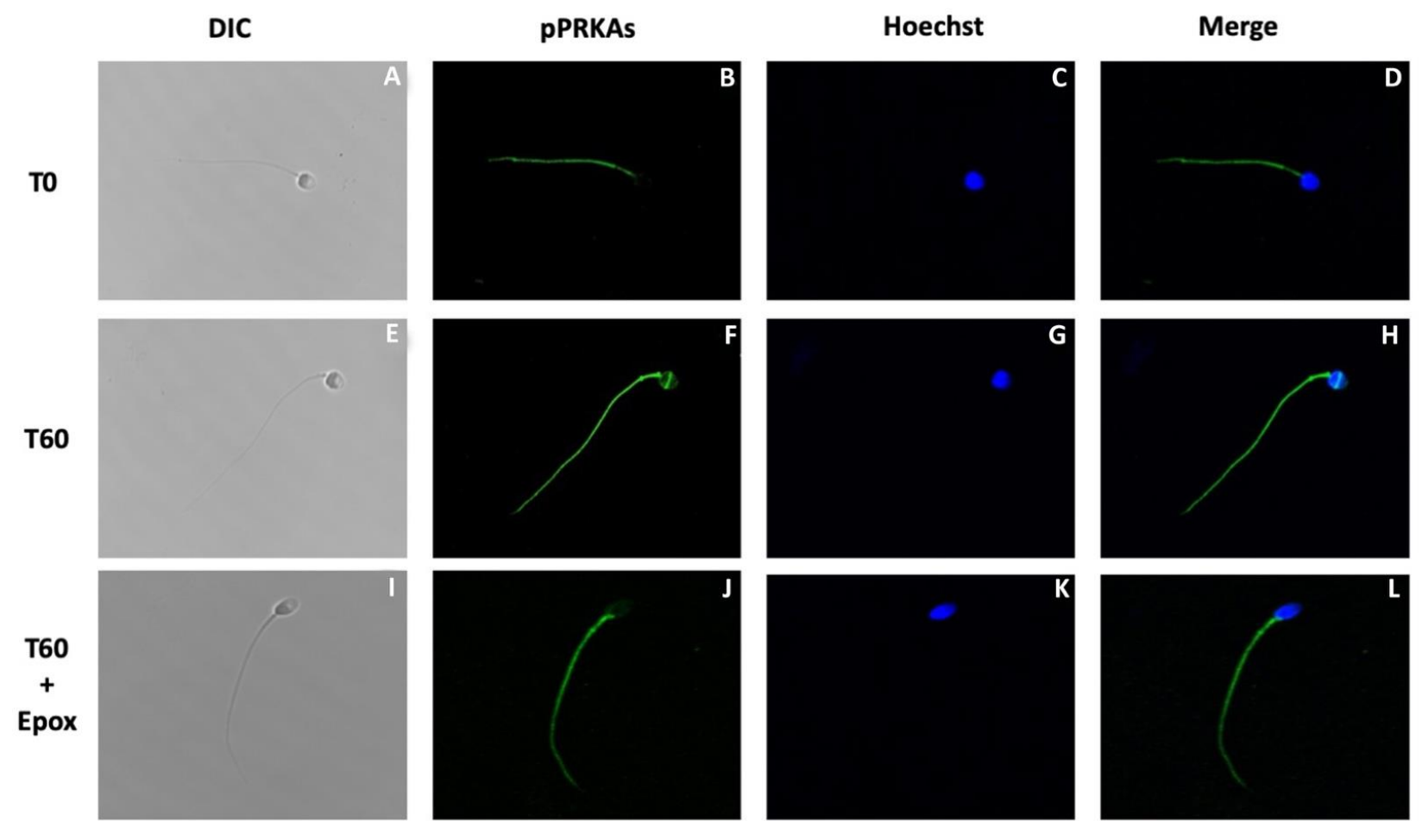

Figure 3. Effect of proteasome inhibition on the localization of phosphorylated PRKA substrates (pPRKAs) during sperm capacitation. Human sperm were incubated for 0 (T0) and 60 (T60) min with $0.1 \%$ DMSO or for 60 min with $10 \mu \mathrm{M}$ epoxomicin (T60 + Epox). Cells were then fixed and labeled with a primary anti-PRKA phosphosubstrates antibody (B,F,J; green) and with Hoechst (C,G,K; blue). DIC: differential interference contrast (A,E,I). Merge: merged image of phosphorylated PRPKA substrates and Hoechst (D,H,L; green and blue).

\subsection{AKAP3 Is Degraded during Sperm Capacitation}

The following experiments were designed to study the possible molecule(s) that could be degraded by the proteasome and that regulate PRKA activity during capacitation. One likely candidate is AKAP3, which was shown to be degraded by the proteasome during bovine sperm capacitation [35]. First, we evaluated the localization of AKAP3 in human sperm (Figure $4 \mathrm{~A}-\mathrm{D})$. The results reveal that most of the spermatozoa (91 $\pm 4 \%$ ) exhibit labeling in the flagellum with a strong mark in the midpiece (Figure 4D). Furthermore, AKAP3 was observed in the sperm head with a strong label in the acrosomal region and in the equatorial line $(96 \pm 3 \%)$.

Next, we performed a capacitation kinetic of the protein levels of AKAP3. AKAP3 protein levels remained constant for $60 \mathrm{~min}$ of incubation in CM (Figure 5A). Subsequently, at 180 and 300 min of incubation, a significant decrease in AKAP3 protein levels was observed (Figure 5B; $p<0.01$ ). At the end of the incubations (180 and $300 \mathrm{~min}$ ), no significant decrease in the motility or viability of the sperm was detected (data not shown), indicating that the decrease in AKAP3 levels was not due to sperm death. Moreover, the protein levels of AKAP3 were evaluated during capacitation in the presence of $10 \mu \mathrm{M}$ epoxomicin (Figure 5A, last right lane). Those sperm that were incubated for $300 \mathrm{~min}$ in 
the presence of epoxomicin did not show a decrease in AKAP3 protein levels (Figure 5B; $p<0.01$ ). These results strongly suggest that the decrease in AKAP3 protein levels was due to proteasome degradation during human sperm capacitation. However, the degradation of AKAP3 started at $180 \mathrm{~min}$, and, taking into account that the effect of the proteasome on PRKA activity was observed after $30 \mathrm{~min}$, this degradation would not explain the regulation of the PRKA activity by the proteasome.
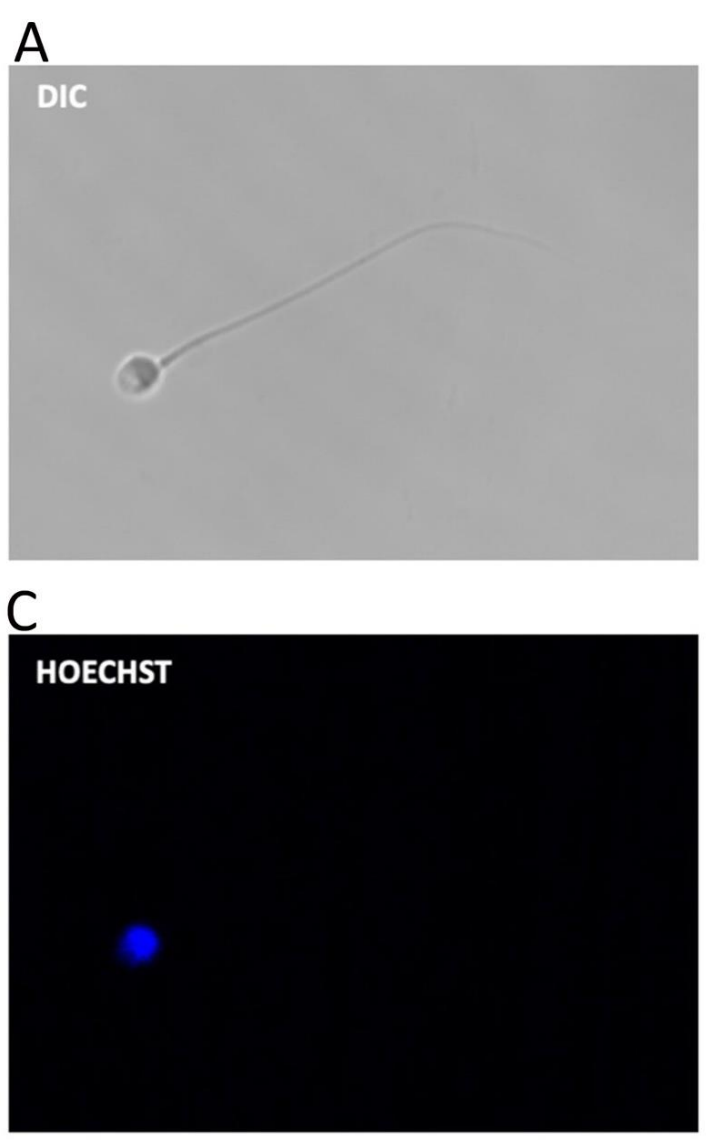
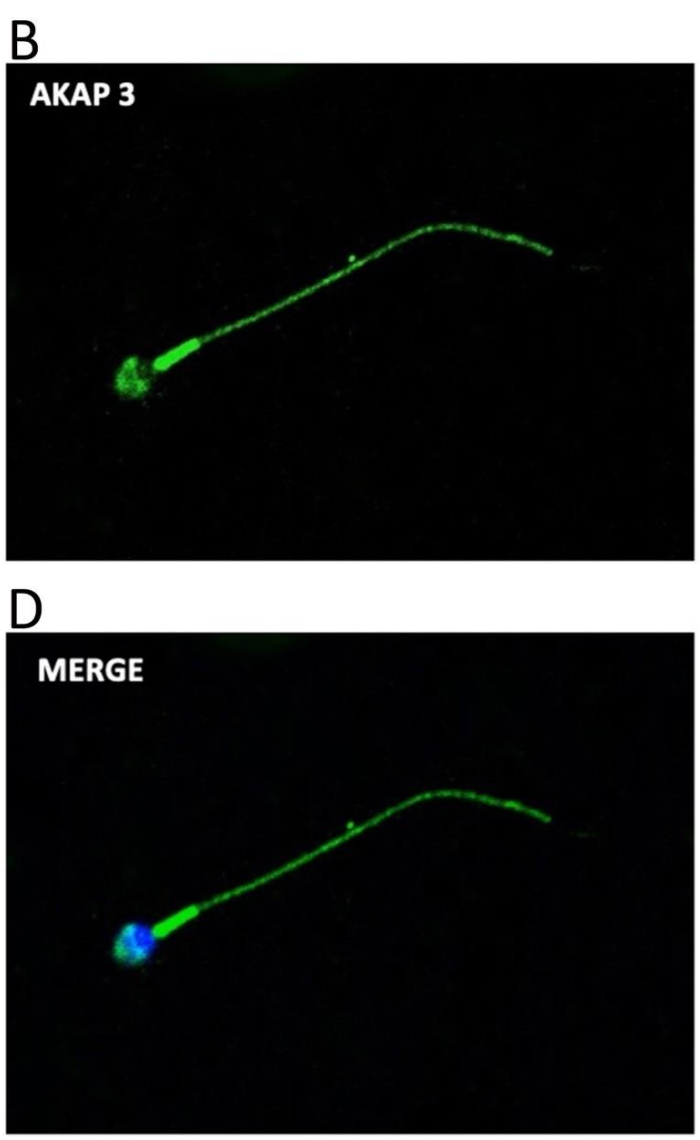

Figure 4. Subcellular location of AKAP3 during human sperm capacitation. Sperm suspensions were incubated under capacitating conditions for $60 \mathrm{~min}$. Then, the cells were fixed and labeled with a primary anti-AKAP3 antibody (B, green) and with Hoechst (C), (blue). DIC: differential interference contrast (A). Merge: merged image of AKAP3 and Hoechst ((D); green and blue).

\subsection{PRKAR1 Is Degraded during Sperm Capacitation}

PRKA is composed of two catalytic subunits (PRKAC) associated with two regulatory subunits (PRKAR1 and PRKAR2). Considering that the catalytic subunits of PRKA remained inhibited by the interaction with the regulatory subunits, the next step was to evaluate whether these regulatory subunits were degraded by the proteasome. The results showed that a molecular weight band of $52 \mathrm{kDa}$ was observed at the different capacitation times that correspond to the PRKAR2 protein. This band did not show significant variations during 60 min incubation in CM (Figure 6A). Regarding its location, PRKAR2 is located only in the flagellum $(88 \pm 8 \%$ ) with a strong intensity in the middle piece (Figure 6D). None of the analyzed sperm had a mark on the head. 

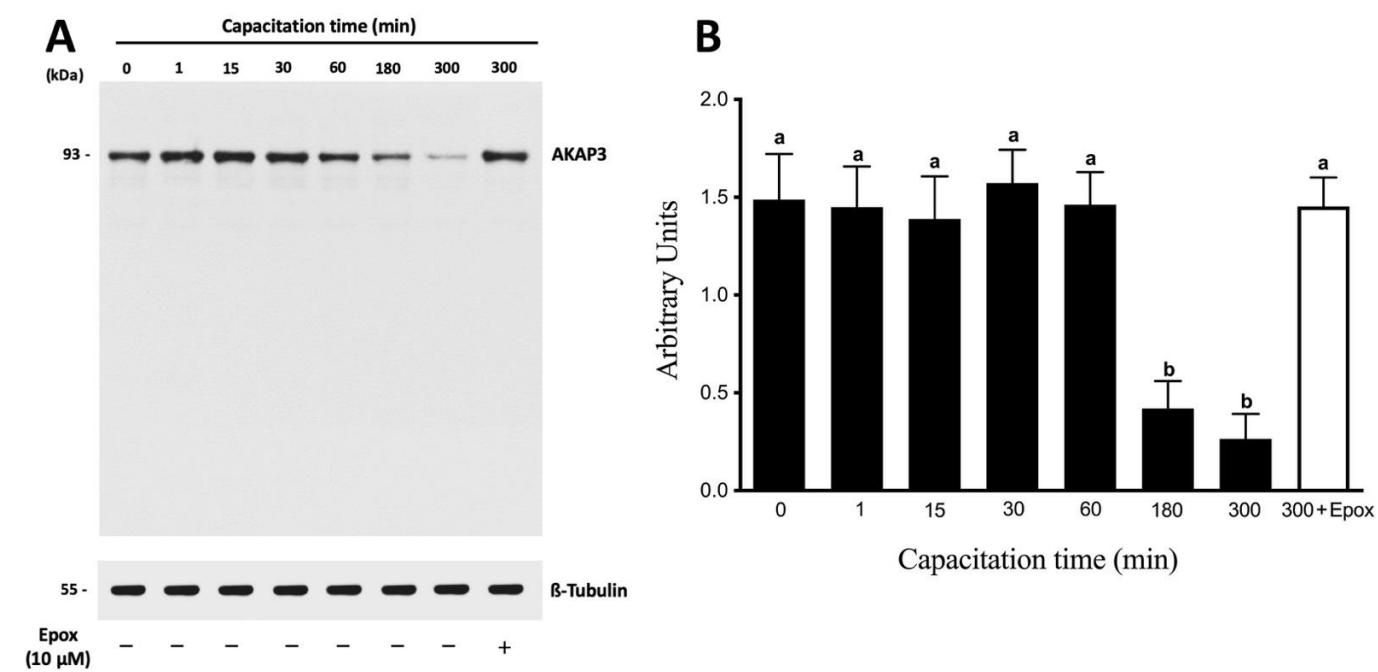

Figure 5. The degradation of AKAP3 is dependent on the sperm proteasome. Total protein extracts were obtained from sperm incubated at different times with $0.1 \%$ DMSO or for 300 min with $10 \mu \mathrm{M}$ epoxomicin (Epox). (A) The level of AKAP3 was detected by Western blot using an anti-AKAP3 antibody. (B) For the densitometric analysis, $\beta$-tubulin was used as a loading control. Bars represent the mean \pm S.E.M of five different experiments. Different letters indicate statistically significant differences $(p<0.01)$ between the groups.

\section{A}

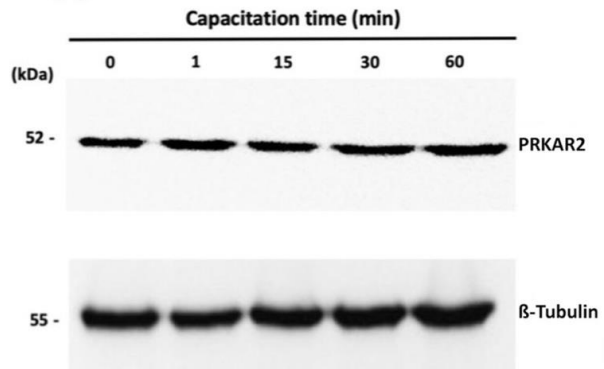

C

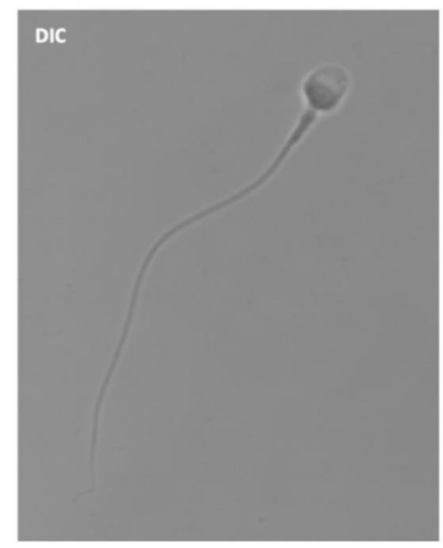

B

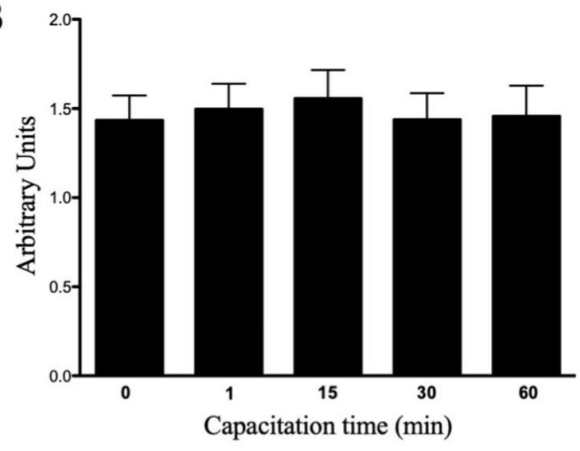

D

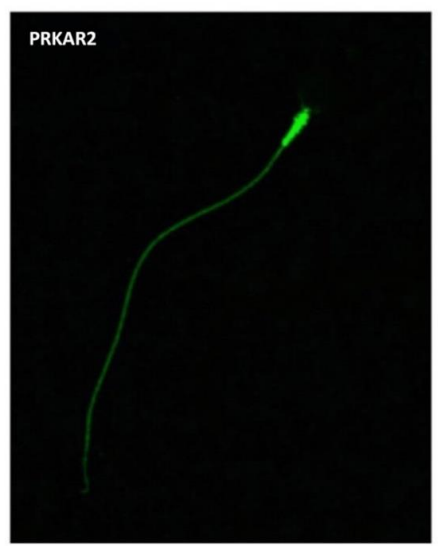

Figure 6. Protein levels and subcellular localization of PRKAR2 during human sperm capacitation. (A) Total protein extracts were obtained from sperm incubated for $0,1,15,30$, or $60 \mathrm{~min}$. The level of PRKAR2 was detected by Western blot using an anti-PRKAR2 antibody. (B) For the densitometric analysis, $\beta$-tubulin was used as a loading control. Bars represent the mean \pm S.E.M of six different experiments. (C,D) For subcellular localization, the sperm were incubated under capacitated conditions for $1 \mathrm{~min}$. Then, the cells were fixed and labeled with a primary anti-PRKAR2 antibody (D, green). DIC: differential interference contrast (C). 
Next, we evaluated the protein levels of PRKAR1. A $48 \mathrm{kDa}$ molecular weight band corresponding to PRKAR1 was observed (Figure 7A). The results showed that PRKAR1 did not present significant variations in their protein levels up to $15 \mathrm{~min}$ of incubation in a capacitating medium (Figure 7B). As can be seen, protein levels of PRKAR1 began to decrease significantly after 30 min of capacitation (Figure 7B; $p<0.01$ ). However, in three out of nine experiments with different donors, PRKAR1 protein levels began to decrease after $60 \mathrm{~min}$ (data not shown). At the end of the incubation, we could not detect any significant decrease in sperm motility and viability, demonstrating that the decrease in PRKAR1 levels was not due to cell death. We then investigated whether the decrease in PRKARI levels was mediated by proteasomal activity. In fact, the decrease in PRKAR1 after $60 \mathrm{~min}$ of incubation in capacitating conditions was significantly inhibited by the presence of epoxomicin (Figure 7A, last right lane; Figure 7B; $p<0.01$ ), indicating that the degradation of PRKAR1 occurred through the proteasomal machinery.
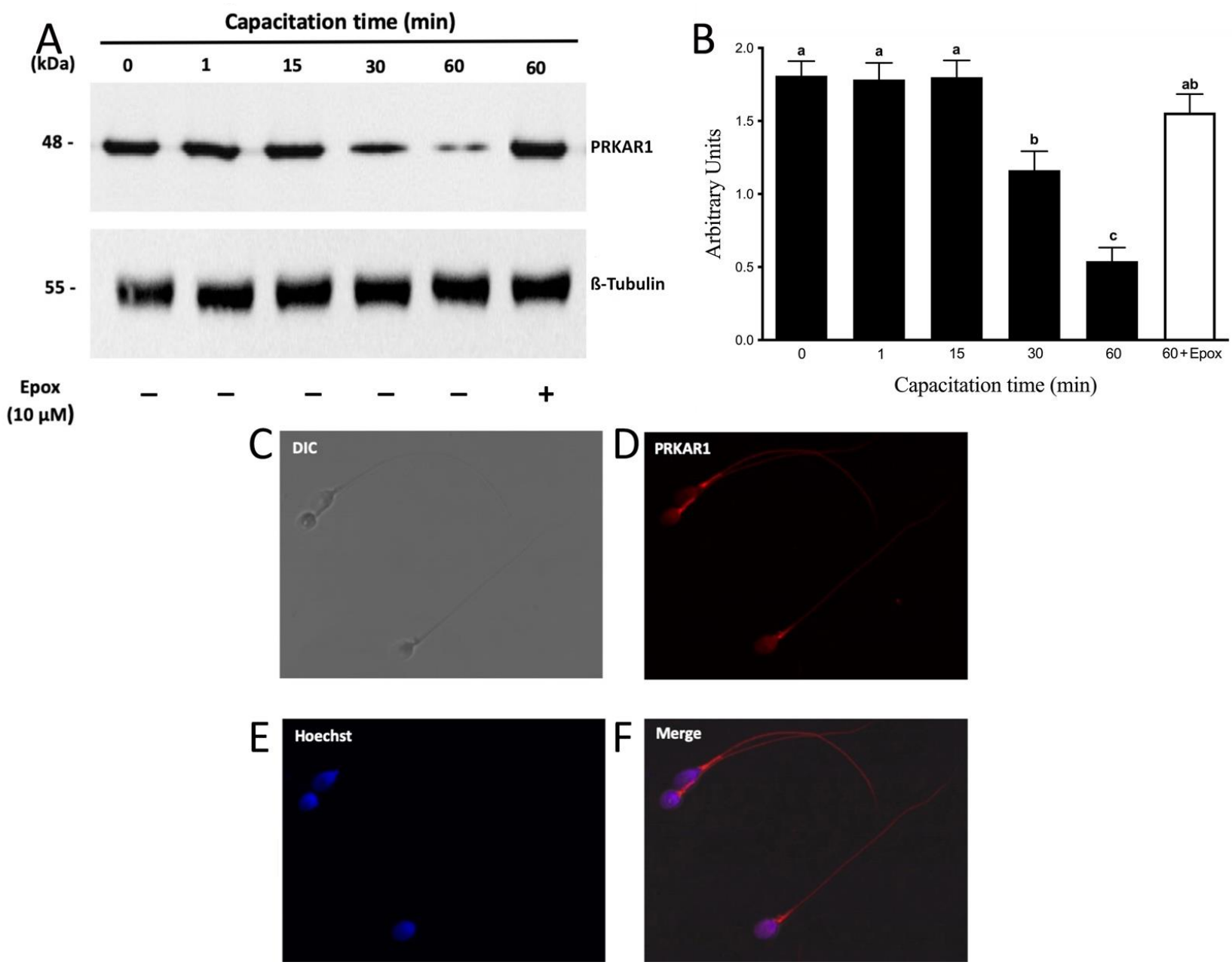

Figure 7. The degradation of PRKAR1 is dependent on the sperm proteasome. Total protein extracts were obtained from sperm incubated at different times with $0.1 \%$ DMSO or for 60 min with $10 \mu \mathrm{M}$ epoxomicin (Epox). (A) The level of PRKAR1 was detected by Western blot using an anti-PRKAR1 antibody. (B) For the densitometric analysis, $\beta$-tubulin was used as a loading control. Bars represent the mean \pm S.E.M of seven different experiments. Different letters indicate statistically significant differences $(p<0.01)$ between groups. $(\mathbf{C}-\mathbf{F})$ For subcellular localization of PRKAR1, sperm were incubated under capacitation conditions for $1 \mathrm{~min}$. Then, the cells were fixed and labeled with a primary anti-PRKARI antibody (D, red) and with Hoechst (E, blue). DIC: differential interference contrast (A). Merge: merged image of PRKAR1 and Hoechst (F; red and blue). 
When evaluating the location of PRKAR1 (Figure 7C-F), it was observed that this protein was located both in the flagellum and in the head of human sperm (92 $\pm 5 \%)$, which is consistent with proteasomal location in these cells (Figure 7F).

\section{Discussion}

Proteasomes play important roles during sperm capacitation [16-18]. In the present study, we show that the proteasome degrades PRKAR1 in human sperm. This is based on the finding that the proteasomal inhibitor epoxomicin significantly inhibited the decrease in PRKAR1 levels. Degradation of PRKAR1 by the proteasome could explain the maintenance of PRKA activity in spite of low intracellular levels of cAMP during sperm capacitation. It is well accepted that the reproductive success of mammalian sperm requires precise orchestration of multiple events, all of them regulated through the cAMP-dependent PRKA pathway. PRKA activation is a necessary element for sperm capacitation; sperm from animals lacking the PRKAC subunit are infertile, despite normal mating behavior [23]. In the present study, we report that PRKA activity increased rapidly one minute after the start of capacitation and then remained high until $60 \mathrm{~min}$ of capacitation compared to spermatozoa incubated in non-capacitating conditions. These results are consistent with those in prior published work [26,27]. The activation of PRKA was followed by an increase in pPRKAs. The results obtained with the antibody that recognizes pPRKAs showed an immediate increase after $1 \mathrm{~min}$ of sperm capacitation, similar to that described by other authors $[28,36]$. The increase in PRKA activity reached its peak after $5 \mathrm{~min}$ and remained constant up to $60 \mathrm{~min}$. This constant phosphorylation observed up to $60 \mathrm{~min}$ of capacitation is consistent with that described by Battistone et al. [28]. The phosphorylated bands detected with the anti pPRKAs antibody during capacitation were dependent on PRKA activity, since in the presence of a PRKA inhibitor the bands did not increase and there were no differences with spermatozoa incubated in NCM. Regarding the subcellular localization of pPRKAs in human sperm, pPRKAs were detected at the flagellar level in non-capacitated and capacitated sperm. However, a higher intensity of fluorescence was obtained at the flagellar level in capacitated spermatozoa. Several studies have shown that during capacitation, flagellar proteins are phosphorylated in tyrosine residues and that this step is necessary for the acquisition of hyperactive motility [37]. Considering that in sperm, PRKA phosphorylates various proteins in Ser and Thr residues to finally activate a signaling cascade leading to increased phosphorylation of proteins in tyrosine residues [38], it is correct to assume that this Tyr phosphorylation depends on PRKA activity. Battistone et al. [28] described that pPRKAs localize only at the flagellar level with no variation during capacitation. However, our results indicate that $24 \pm 6 \%$ of the capacitated sperm have a mark in the equatorial segment of the head. This localization of pPRKAs is consistent with the localization of PRKA [16,39-41], AKAPs [42], and with phosphorylation in Ser and Thr residues, described at the level of the head by Naz [43] in human spermatozoa.

In our study, the inhibition of proteasome activity early during capacitation decreased phosphorylation and changed the subcellular localization of pPRKAs in human sperm. Something similar has been described in later events of capacitation. Human sperm incubated for $18 \mathrm{~h}$ in the presence of proteasome inhibitors exhibited changes in the protein phosphorylation pattern at Ser and Thr residues [44]. When evaluating the activity of PRKA, it increased significantly after $1 \mathrm{~min}$ of capacitation, remaining high throughout the incubation. However, epoxomicin treatment significantly decreased PRKA activity after $30 \mathrm{~min}$ of capacitation. These results indicate that the proteasome regulates the maintenance of PRKA activity during early capacitation. As a precedent, PRKA activity is regulated by the proteasome in other cellular systems. In Aplysia, the proteasome has been shown to regulate PRKA activity during synaptic plasticity [45]. This has also been shown in neuroblastoma cells, where the proteasome regulates the maintenance of PRKA activity during long-term memory [46]. Our group recently published that during the early capacitation of human sperm, the activity of the proteasome increases after $5 \mathrm{~min}$ of capacitation and is regulated by the SACY/cAMP/PRKA pathway [16]. In this new 
study, we present evidence that the maintenance of PRKA activity is regulated by the proteasome after $30 \mathrm{~min}$ of capacitation, which leads to the regulation of pPRKAs. Given this background, we will now discuss the possible mechanism of PRKA activity regulation by the proteasome during early events of capacitation.

The activation of PRKA depends on its adequate localization in subcellular regions. AKAP proteins are responsible for targeting PRKA and other proteins to specific subcellular locations [47]. AKAPs play important roles in sperm function, including capacitation and acrosome reaction [48]. Hillman et al. [35] demonstrated that AKAP3 is degraded by the proteasome after $4 \mathrm{~h}$ of capacitation in bovine sperm. Furthermore, Vizel et al. [48] demonstrated that this degradation is important for capacitation to occur in bovine sperm. Here, we present evidence that AKAP3 is degraded by the proteasome during human sperm capacitation. However, the degradation of AKAP3 occurs after $3 \mathrm{~h}$ of capacitation. Thus, this degradation does not explain the maintenance of PRKA activity by the proteasome during capacitation, an effect that was observed after $30 \mathrm{~min}$. This degradation of AKAP3 may be related to the acrosomal reaction that corresponds to a post-capacitation event. In human sperm, it has been shown that inhibition of the proteasome decreases the percentage of sperm that undergo the acrosomal reaction [44] and that PRKA plays a role during this event [49].

PRKA is a tetrameric enzyme consisting of two catalytic subunits (PRKAC), which are maintained in an inactive state by binding to regulatory subunits (PRKAR). In mice, the elimination of PRKAR2 improves the basal activity of PRKAC, leading to an increase in the metabolic rate in adipose tissue [50]. In other cellular models, regulatory subunits have been shown to be degraded by the proteasome. In Aplysia sensory neurons, PRKAR is degraded by the proteasome, with consequent persistent activation of PRKAC during synaptic plasticity [45]. Lignitto et al. [46], demonstrated that the PRKAR subunit is ubiquitinated, which allows the proteasomal degradation of this subunit. The ubiquitination of PRKAR is through a signaling mechanism involving cAMP/PRKA. Furthermore, they demonstrated that this degradation is necessary to maintain high PRKAC activity in long-term memory processes [46] through a positive feedback mechanism between PRKA and the proteasome.

In this study, we report by immunofluorescence analysis that PRKAR2 is located exclusively at the flagellar level, while PRKAR1 is located both at the flagellar level and in the head. These results are similar to those described in sperm from other species [51,52]. This differential location could be related to different roles for the PRKAR1 and PRKAR2 isoforms in sperm function. When evaluating the protein levels of these subunits, we observed that the PRKAR2 remained constant during incubation, suggesting that this subunit is not degraded in the early stages of capacitation. This correlates with the subcellular location of PRKAR2, since the proteasome in human sperm is not located at the flagellar level $[14,16,53,54]$. On the other hand, protein levels of PRKAR1 in human sperm decreased after capacitation for $30 \mathrm{~min}$. This decrease in PRKAR1 levels was most probably due to the degradation of this subunit by the proteasome, since this process was inhibited by epoxomicin. In conclusion, our results show that the maintenance of PRKA activity is regulated by the proteasome after $30 \mathrm{~min}$ of capacitation, which is correlated with the degradation time of PRKAR1. These results indicate that PRKAR1 is a possible target of proteasome regulation of the maintenance of PRKA activity during early human sperm capacitation. Additional studies are needed to fully understand the relationship between PRKAR1 and the proteasome in mammalian sperm.

Supplementary Materials: The following is available online at https:/ /www.mdpi.com/article/10 .3390/cells10123501/s1, Table S1: Semen quality parameters of ejaculates used in the study.

Author Contributions: Conceptualization, H.Z.-C., M.K., and P.M.; methodology and validation, H.Z.-C., L.B., M.K., and P.M.; formal analysis, H.Z.-C., L.B., and P.M.; writing-original draft preparation, H.Z.-C. and M.K.; writing-review and editing H.Z.-C. and P.M.; project administration and funding acquisition, P.M. All authors have read and agreed to the published version of the manuscript. 
Funding: This research was funded by Fondo Puente de lnvestigación de Excelencia of the University of Antofagasta CR-4496 (PM).

Institutional Review Board Statement: The study was conducted according to the guidelines of the Declaration of Helsinki, and approved by the Ethics Committee on Scientific Research of the University of Antofagasta (CEIC-UA). Approved protocol (\#143 approved on 2018).

Informed Consent Statement: Informed consent was obtained from all subjects involved in the study.

Data Availability Statement: Not applicable.

Conflicts of Interest: The authors declare no conflict of interest.

\section{References}

1. Schwartz, A.L.; Ciechanover, A. Targeting proteins for destruction by the ubiquitin system: Implications for human pathobiology. Annu. Rev. Pharmacol. Toxicol. 2009, 49, 73-96. [CrossRef]

2. Meyer-Schwesinger, C. The ubiquitin-proteasome system in kidney physiology and disease. Nat. Rev. Nephrol. 2019, 15, 393-411. [CrossRef] [PubMed]

3. Schlesinger, D.H.; Goldstein, G.; Niall, H.D. The complete amino acid sequence of ubiquitin, an adenylate cyclase stimulating polypeptide probably universal in living cells. Biochemistry 1975, 14, 2214-2218. [CrossRef]

4. Bebington, C.; Doherty, F.J.; Fleming, S.D. The possible biological and reproductive functions of ubiquitin. Hum. Reprod. Update 2001, 7, 102-111. [CrossRef]

5. Ciechanover, a Intracellular protein degradation: From a vague idea thru the lysosome and the ubiquitin-proteasome system and onto human diseases and drug targeting. Cell Death Differ. 2005, 12, 1178-1190. [CrossRef] [PubMed]

6. Heinemeyer, W.; Kleinschmidt, J.A.; Saidowsky, J.; Escher, C.; Wolf, D.H. Proteinase yscE, the yeast proteasome/multicatalyticmultifunctional proteinase: Mutants unravel its function in stress induced proteolysis and uncover its necessity for cell survival. EMBO J. 1991, 10, 555-562. [CrossRef] [PubMed]

7. Velichutina, I.; Connerly, P.L.; Arendt, C.S.; Li, X.; Hochstrasser, M. Plasticity in eucaryotic $20 \mathrm{~S}$ proteasome ring assembly revealed by a subunit deletion in yeast. EMBO J. 2004, 23, 500-510. [CrossRef]

8. Jung, T.; Catalgol, B.; Grune, T. The proteasomal system. Mol. Asp. Med. 2009, 30, 191-296. [CrossRef]

9. Adams, J. The development of proteasome inhibitors as anticancer drugs. Cancer Cell 2004, 5, 417-421. [CrossRef]

10. Jung, T.; Grune, T. Structure of the proteasome. Prog. Mol. Biol. Transl. Sci. 2012, 109, 1-39. [CrossRef]

11. Bousquet-Dubouch, M.-P.; Fabre, B.; Monsarrat, B.; Burlet-Schiltz, O. Proteomics to study the diversity and dynamics of proteasome complexes: From fundamentals to the clinic. Expert Rev. Proteom. 2011, 8, 459-481. [CrossRef] [PubMed]

12. Thibaudeau, T.A.; Smith, D.M. A practical review of proteasome pharmacology. Pharmacol. Rev. 2019, 71, 170-197. [CrossRef]

13. Tipler, C.P.; Hutchon, S.P.; Hendil, K.; Tanaka, K.; Fishel, S.; Mayer, R.J. Purification and characterization of $26 \mathrm{~S}$ proteasomes from human and mouse spermatozoa. Mol. Hum. Reprod. 1997, 3, 1053-1060. [CrossRef]

14. Wojcik, C.; Benchaib, M.; Lornage, J.; Czyba, J.C.; Guerin, J.F. Proteasomes in human spermatozoa. Int. J. Androl. 2000, $23,169-177$. [CrossRef] [PubMed]

15. Morales, P.; Kong, M.; Pizarro, E.; Pasten, C. Participation of the sperm proteasome in human fertilization. Hum. Reprod. 2003, 18, 1010-1017. [CrossRef]

16. Zapata-Carmona, H.; Barón, L.; Zuñiga, L.M.; Díaz, E.S.; Kong, M.; Drobnis, E.Z.; Sutovsky, P.; Morales, P. The activation of the chymotrypsin-like activity of the proteasome is regulated by soluble adenyl cyclase/cAMP/protein kinase A pathway and required for human sperm capacitation. Mol. Hum. Reprod. 2019, 25, 587-600. [CrossRef] [PubMed]

17. Kerns, K.; Morales, P.; Sutovsky, P. Regulation of Sperm Capacitation by the 26S Proteasome: An Emerging New Paradigm in Spermatology. Biol. Reprod. 2016, 94, 117. [CrossRef]

18. Zigo, M.; Manaskova-Postlerova, P.; Jonakova, V.; Kerns, K.; Sutovsky, P. Compartmentalization of the proteasome-interacting proteins during sperm capacitation. Sci. Rep. 2019, 9, 12583. [CrossRef]

19. Austin, C.R. The capacitation of the mammalian sperm. Nature 1952, 170, 326. [CrossRef]

20. Chang, M.C. Fertilizing capacity of spermatozoa deposited into the fallopian tubes. Nature 1951, 168, 697-698. [CrossRef]

21. Zapata-Carmona, H.; Soriano-Úbeda, C.; París-Oller, E.; Matás, C. Periovulatory oviductal fluid decreases sperm protein kinase A activity, tyrosine phosphorylation, and in vitro fertilization in pig. Andrology 2020, 8, 756-768. [CrossRef] [PubMed]

22. Finkelstein, M.; Etkovitz, N.; Breitbart, H. Ca ${ }^{2+}$ Signaling in Mammalian Spermatozoa. Mol. Cell Endocrinol. 2020, $516,110953$. [CrossRef] [PubMed]

23. Nolan, M.A.; Babcock, D.F.; Wennemuth, G.; Brown, W.; Burton, K.A.; McKnight, G.S. Sperm-specific protein kinase A catalytic subunit Calpha2 orchestrates cAMP signaling for male fertility. Proc. Natl. Acad. Sci. USA 2004, 101, 13483-13488. [CrossRef] [PubMed]

24. Shabb, J.B. Physiological substrates of cAMP-dependent protein kinase. Chem. Rev. 2001, 101, 2381-2411. [CrossRef]

25. Bruce, J.I.E.; Shuttleworth, T.J.; Giovannucci, D.R.; Yule, D.I. Phosphorylation of inositol 1,4,5-trisphosphate receptors in parotid acinar cells: A mechanism for the synergistic effects of cAMP on $\mathrm{Ca}^{2+}$ signaling. J. Biol. Chem. 2002, 277, 1340-1348. [CrossRef] [PubMed] 
26. Moseley, F.L.C.; Jha, K.N.; Björndahl, L.; Brewis, I.A.; Publicover, S.J.; Barratt, C.L.R.; Lefièvre, L. Protein tyrosine phosphorylation, hyperactivation and progesterone-induced acrosome reaction are enhanced in IVF media: An effect that is not associated with an increase in protein kinase A activation. Mol. Hum. Reprod. 2005, 11, 523-529. [CrossRef]

27. Martínez-León, E.; Osycka-Salut, C.; Signorelli, J.; Pozo, P.; Pérez, B.; Kong, M.; Morales, P.; Pérez-Martínez, S.; Díaz, E.S. Fibronectin stimulates human sperm capacitation through the cyclic AMP/protein kinase A pathway. Hum. Reprod. 2015, 30, 2138-2151. [CrossRef]

28. Battistone, M.A.; Da Ros, V.G.; Salicioni, A.M.; Navarrete, F.A.; Krapf, D.; Visconti, P.E.; Cuasnicú, P.S. Functional human sperm capacitation requires both bicarbonate-dependent PKA activation and down-regulation of Ser/Thr phosphatases by Src family kinases. Mol. Hum. Reprod. 2013, 19, 570-580. [CrossRef]

29. Björndahl, L.; Barratt, C.L.R.; Mortimer, D.; Jouannet, P. “How to count sperm properly": Checklist for acceptability of studies based on human semen analysis. Hum. Reprod. 2016, 31, 227-232. [CrossRef]

30. WHO World Health Orgnization. WHO Laboratory Manual for the Examination and Processing of Human Semen, 5th ed.; WHO World Health Orgnization: Geneva, Switzerland, 2010; ISBN 9789241547789.

31. Laemmli, U.K. Cleavage of structural proteins during the assembly of the head of bacteriophage T4. Nature 1970, 227, 680-685. [CrossRef]

32. Jin, H.X.; Wu, T.X.; Jiang, Y.J.; Zou, J.W.; Zhuang, S.L.; Mao, X.; Yu, Q. Sen Role of phosphorylated Thr-197 in the catalytic subunit of cAMP-dependent protein kinase. J. Mol. Struct. THEOCHEM 2007, 805, 9-15. [CrossRef]

33. Pearce, L.R.; Komander, D.; Alessi, D.R. The nuts and bolts of AGC protein kinases. Nat. Rev. Mol. Cell Biol. 2010, 11, 9-22. [CrossRef]

34. Moore, M.J.; Kanter, J.R.; Jones, K.C.; Taylor, S.S. Phosphorylation of the catalytic subunit of protein kinase A: Autophosphorylation versus phosphorylation by phosphoinositide-dependent kinase-1. J. Biol. Chem. 2002, 277, 47878-47884. [CrossRef]

35. Hillman, P.; Ickowicz, D.; Vizel, R.; Breitbart, H. Dissociation between AKAP3 and PKARII Promotes AKAP3 Degradation in Sperm Capacitation. PLoS ONE 2013, 8, e68873. [CrossRef]

36. O'Flaherty, C.; de Lamirande, E.; Gagnon, C. Phosphorylation of the Arginine-X-X-(Serine/Threonine) motif in human sperm proteins during capacitation: Modulation and protein kinase A dependency. Mol. Hum. Reprod. 2004, 10, 355-363. [CrossRef]

37. Nassar, A.; Mahony, M.; Morshedi, M.; Lin, M.H.; Srisombut, C.; Oehninger, S. Modulation of sperm tail protein tyrosine phosphorylation by pentoxifylline and its correlation with hyperactivated motility. Fertil. Steril. 1999, 71, 919-923. [CrossRef]

38. Visconti, P.E. Understanding the molecular basis of sperm capacitation through kinase design. Proc. Natl. Acad. Sci. USA 2009, 106, 667-668. [CrossRef]

39. Neuhaus, E.M.; Mashukova, A.; Barbour, J.; Wolters, D.; Hatt, H. Novel function of $\beta$-arrestin2 in the nucleus of mature spermatozoa. J. Cell Sci. 2006, 119, 3047-3056. [CrossRef]

40. Mitchell, L.A.; Nixon, B.; Baker, M.A.; Aitken, R.J. Investigation of the role of SRC in capacitation-associated tyrosine phosphorylation of human spermatozoa. MHR Basic Sci. Reprod. Med. 2008, 14, 235-243. [CrossRef]

41. Vetter, M.M.; Zenn, H.M.; Méndez, E.; Van Den Boom, H.; Herberg, F.W.; Skålhegg, B.S. The testis-specific C $\alpha 2$ subunit of PKA is kinetically indistinguishable from the common C1 subunit of PKA. BMC Biochem. 2011, 12, 40. [CrossRef] [PubMed]

42. Harrison, D.A.; Carr, D.W.; Meizel, S. Involvement of Protein Kinase A and A Kinase Anchoring Protein in the ProgesteroneInitiated Human Sperm Acrosome Reaction. Biol. Reprod. 2000, 820, 811-820. [CrossRef] [PubMed]

43. Naz, R.K. Involvement of protein serine and threonine phosphorylation in human sperm capacitation. Biol. Reprod. 1999, 60, 1402-1409. [CrossRef]

44. Kong, M.; Diaz, E.S.; Morales, P. Participation of the human sperm proteasome in the capacitation process and its regulation by protein kinase A and tyrosine kinase. Biol. Reprod. 2009, 80, 1026-1035. [CrossRef]

45. Hegde, A.N. Ubiquitin-proteasome-mediated local protein degradation and synaptic plasticity. Prog. Neurobiol. 2004, 73, 311-357. [CrossRef] [PubMed]

46. Lignitto, L.; Carlucci, A.; Sepe, M.; Stefan, E.; Cuomo, O.; Nisticò, R.; Scorziello, A.; Savoia, C.; Garbi, C.; Annunziato, L.; et al. Control of PKA stability and signalling by the RING ligase praja2. Nat. Cell Biol. 2011, 13, 412-422. [CrossRef]

47. Scott, J.D.; Dell'Acqua, M.L.; Fraser, I.D.C.; Tavalin, S.J.; Lester, L.B. Coordination of cAMP Signaling Events through PKA Anchoring. Adv. Pharmacol. 1999, 47, 175-207. [CrossRef]

48. Vizel, R.; Hillman, P.; Ickowicz, D.; Breitbart, H. AKAP3 degradation in sperm capacitation is regulated by its tyrosine phosphorylation. Biochim. Biophys. Acta-Gen. Subj. 2015, 1850, 1912-1920. [CrossRef] [PubMed]

49. Barón, L.; Fara, K.; Zapata-Carmona, H.; Zuñiga, L.; Kong, M.; Signorelli, J.; Díaz, E.S.; Morales, P. Participation of protein kinases and phosphatases in the progesterone-induced acrosome reaction and calcium influx in human spermatozoa. Andrology 2016, 4, 1073-1083. [CrossRef] [PubMed]

50. Newhall, K.J.; Cummings, D.E.; Nolan, M.A.; McKnight, G.S. Deletion of the RII $\beta$-subunit of protein kinase A decreases body weight and increases energy expenditure in the obese, leptin-deficient ob/ob mouse. Mol. Endocrinol. 2005, 19, 982-991. [CrossRef] [PubMed]

51. Vijayaraghavan, S.; Liberty, G.A.; Mohan, J.; Winfrey, V.P.; Olson, G.E.; Carr, D.W. Isolation and molecular characterization of AKAP110, a novel, sperm-specific protein kinase A-anchoring protein. Mol. Endocrinol. 1999, 5, 705-717. [CrossRef] 
52. Vijayaraghavan, S.; Olson, G.E.; NagDas, S.; Winfrey, V.P.; Carr, D.W. Subcellular localization of the regulatory subunits of cyclic adenosine 3',5'-monophosphate-dependent protein kinase in bovine spermatozoa. Biol. Reprod. 1997, 57, 15171523. [CrossRef] [PubMed]

53. Biały, L.P.; Ziemba, H.T.; Marianowski, P.; Fracki, S.; Bury, M.; Wójcik, C. Localization of a proteasomal antigen in human spermatozoa: Immunohistochemical electron microscopic study. Folia Histochem. Cytobiol. 2001, 39, 129-130. [PubMed]

54. Morales, P.; Pizarro, E.; Kong, M.; Jara, M. Extracellular localization of proteasomes in human sperm. Mol. Reprod. Dev. 2004, 68, 115-124. [CrossRef] [PubMed] 